\title{
openheart Body composition in heart failure and the impact of cardiac resynchronisation therapy: a proof-of-concept study
}

\author{
Christopher J McAloon, ${ }^{1,2}$ Samantha Hyndman, ${ }^{1}$ Valerie Ansell, ${ }^{1}$ Paul O'Hare, ${ }^{2}$ \\ Harpal Randeva, ${ }^{2}$ Faizel Osman (D) ${ }^{1,2}$
}

\begin{abstract}
- Additional material is published online only. To view please visit the journal online (http://dx.doi.org/10.1136/ openhrt-2019-001105).
\end{abstract}

To cite: McAloon CJ, Hyndman S, Ansell V, et al. Body composition in heart failure and the impact of cardiac resynchronisation therapy: a proof-of-concept study. Open Heart 2020;7:e001105. doi:10.1136/

openhrt-2019-001105

Received 6 June 2019 Revised 7 January 2020 Accepted 7 January 2020
A) Check for updates

(C) Author(s) (or their employer(s)) 2020. Re-use permitted under CC BY-NC. No commercial re-use. See rights and permissions. Published by BMJ.

${ }^{1}$ Cardiology, University Hospital Coventry, Coventry, UK ${ }^{2}$ University of Warwick Warwick Medical School, Coventry, UK

Correspondence to Professor Faizel 0sman; faizel. osman@uhcw.nhs.uk

\section{ABSTRACT}

Aims Body composition (BC) is known to alter in heart failure. Cardiac resynchronisation therapy (CRT) improves left ventricular geometry but the impact on $\mathrm{BC}$ is unknown. Our aim was to evaluate $\mathrm{BC}$ in these patients before and after CRT implantation.

Methods Prospective proof-of-concept pilot study of heart failure patients undergoing CRT between September 2014 and December 2015. Assessments performed preCRT and post-CRT (6 weeks and 6 months) were: BC parameters (using air-displacement plethysmography), New York Heart Failure classification for assessing symptom severity, echocardiography to assess left ventricular geometry, electrocardiography, Minnesota Heart Failure Questionnaire and N-terminal probrain natriuretic peptide (NT-pro-BNP). Repeated measures analysis of variance was performed to assess relative change over time and potential correlations.

Results Twenty-five patients were recruited; meanage $( \pm$ SD) was $73.4 \pm 10.0$ years, 23 males, 18 CRT defibrillators (remainder CRT pacemakers), 16 had ischaemic aetiology, 6 diabetics, 17 with left bundlebranch morphology on ECG and 10 had atrial fibrillation. Significant inverse correlations were observed in the first 6 weeks following CRT between fat mass and left ventricular end-diastolic volume $(r=-0.69, p<0.01)$ and NT-pro-BNP and fat mass $(r=0.41, p=0.05)$. No significant differences were noted over 6 months. There was an observed trend towards reduced fat mass in the first 6 weeks post-CRT implant driven by non-responders. There was no significant difference between responders and non-responders in BC over 6 months.

Conclusion This is the first study to observe interplay between BC and cardiac geometry/function following CRT; a trend in overall fat mass reduction was noted following CRT and merits further study.

\section{INTRODUCTION}

There is a complex interplay between heart failure (HF), body composition and metabolism. ${ }^{1}$ Development of HF causes neurohormonal activation, a proinflammatory state and endothelial dysfunction favouring a procatabolic state, ${ }^{23}$ which is heavily influenced by body composition. ${ }^{1}$ Obesity makes development of HF more likely, ${ }^{4}$ however,

\section{Key questions}

What is already known about this subject?

- There is a complex interplay between heart failure and body composition with neurohormonal activation and endothelial dysfunction

- Cardiac cachexia affects all body composition components and is driven by a procatabolic state and is a predictor of poor heart failure outcomes.

- Cardiac resynchronisation therapy (CRT) causes reverse cardiac remodelling improving both morbidity and mortality but the impact of CRT on body composition in these patients is unclear.

What does this study add?

- This is the first prospective pilot study to examine body composition parameters both before and after CRT implantation.

- It suggests a trend towards reduction in fat mass in CRT non-responders.

- The association between left ventricular geometry and fat mass relative change following CRT suggests that the increased measured fat mass may be linked to reverse cardiac remodelling.

How might this impact on clinic practice?

- Successful CRT response appears to be linked to maintenance of fat mass status at implant and the neurohormonal system appears integral to this.

- Knowledge of the relationship of body composition and CRT may help better identify those who are likely to benefit from CRT.

the presence of adiposity is protective against HF progression. ${ }^{5}$ This observation has been termed the 'obesity paradox'. Higher adiposity is also inversely related to neurohormonal activation. ${ }^{6}$ Sarcopenia is associated with a proinflammatory state ${ }^{7}$ and increased neurohormonal signalling. ${ }^{6}$ Cardiac cachexia affects all body composition components and is driven by a procatabolic state; it is a predictor of poor HF outcomes. ${ }^{245}$ Cardiac resynchronisation therapy (CRT) causes reverse cardiac remodelling improving both morbidity and mortality. ${ }^{8}$ Cai et at observed being overweight/obese predicted CRT 
response and improved 6-month survival suggesting body composition may be impacted and/or altered by CRT. Baseline body composition parameters may also be predictive of CRT response. The aim of our proofof-concept study was to evaluate body composition in patients with HF both before and after CRT implantation.

\section{METHODS}

\section{Patient population}

We performed a prospective pilot study of consecutive patients with HF undergoing CRT meeting National Institute of Clinical Excellence (TA120) implant criteria $^{10}$ between September 2014 and December 2015. The study was conducted in accordance with the Declaration of Helsinki and all patients provided informed consent. Air displacement plethysmography (ADP) was performed preimplant, 6 weeks and 6 months postimplant. All had New York Heart Failure Assessment (NYHA), 6 min walk test, transthoracic echocardiography, Minnesota Living with HF Questionnaire (MLHFQ), resting 12-lead ECG and blood sampling, including N-terminal probrain natriuretic peptide (NT-pro-BNP). All our elective implants were performed as same-day procedures as reported previously. ${ }^{11}$ All underwent echocardiography (Vivid 7, GE Healthcare, Horten, Norway) for left ventricular (LV) assessment by a nationally accredited operator on the same machine with measurements analysed offline. Whole body ADP (BOD-POD-Life Measurement, Concord, California, USA) reliably and reproducibly measures body composition comparable with traditional methods. ${ }^{12}$ Participants were fasted, rested 2 hours pretest and had height/weight measurements taken. All entered BOD-POD wearing a lycra swim cap and underwear only for measurement standardisation; two serial measurements were averaged. Where measurements varied significantly a third test was performed. The primary outcome was functional response status defined as those who survived, did not undergo heart transplantation and achieved two out of three response criteria $(\downarrow>1$ NYHA, $\uparrow \geq 10 \% 6$ MWT distance, $\downarrow$ MLHFQ score $>5$ ) at 6-month follow-up.

\section{Statistical analysis}

Statistical analysis was performed using SPSS, V.22.0 (IBM). Categorical variables were reported as frequency/ percentages. Categorical data were compared using $\mathrm{X}^{2} /$ Fisher's exact tests. Normally distributed continuous data were reported as mean $\pm \mathrm{SD}$ and analysis performed using independent t-tests. Non-normally distributed data were reported as median (+full range) and compared using Mann-Whitney U test. Variation in continuous variables over three time periods was analysed using either one-way analysis of variance/Friedman test. Mixed between-within subjects analysis of variance was used to compare variation in body composition data in functional responders/ non-responders over 6 months. Pearson (parametric) or Spearman's rank (non-parametric) estimators were performed between change in body composition and functional/echocardiographic and neurohormonal parameters; $\mathrm{p}<0.05$ was considered significant.

\section{RESULTS}

Twenty-seven patients were recruited; 25 had baseline body composition assessment (one unable to enter BODPOD, one had significant erroneous measurements despite repeated attempts). Baseline characteristics are shown in table 1 . Three patients were unable to have 6-month body composition assessment (one death during follow-up and two not well enough for BOD-POD assessment). The first follow-up was at 1.7 months (SD: 0.3 months) and final visit was 5.8 months (SD: 0.5 months) post-CRT. Baseline characteristics were no different between responders versus non-responders, including all body composition parameters.

The effect of CRT on cardiac function and body composition is shown in table 2. There was significant improvement in MLHFQ scores and decrease in QRS duration post CRT-implant. Total and percentage fat mass showed reduction trend 6 months post-CRT with improvement trend in LV ejection fraction) and decrease in LV endsystolic volume (LVESV).

Changes over time in body composition for functional responders and non-responders were analysed. Online supplementary A shows changes in body composition after CRT implantation with no difference between responders versus non-responders and no change in body composition over 6 months. However, a trend for higher relative fat mass at baseline for non-responders and large decrease 6 weeks post-CRT compared with responders was noted. After 6 weeks relative fat mass in non-responders returned to levels similar to baseline at 6 months. Responders had minimal variation over the three observation points and had lower relative fat mass than non-responders. There was a trend towards difference between responders and non-responders over time for fat mass.

Online supplementary B shows the strongest associations between body composition and cardiac function post-CRT implant. A strong inverse correlation was noted between LVESV index and relative fat mass over the first 6 weeks and medium inverse correlation between fat mass and NT-pro-BNP. There was medium association between fat mass and estimated glomerular filtration rate (eGFR), $\mathrm{r}=0.41, \mathrm{p}=0.06$, which trended towards significance at 6 weeks. Long term, the strongest inverse correlation was between fat mass and eGFR. A significant association was also observed between eGFR and fat-free mass $(r=0.47$, $\mathrm{p}=0.04)$ at 6 months post-CRT implant.

\section{DISCUSSION}

This is the first study examining body composition before and after CRT implantation in patients with HF and functional response, with association between fat mass and LV geometry noted post-CRT implant. Short-term changes in 
Table 1 Baseline characteristics of the cohort

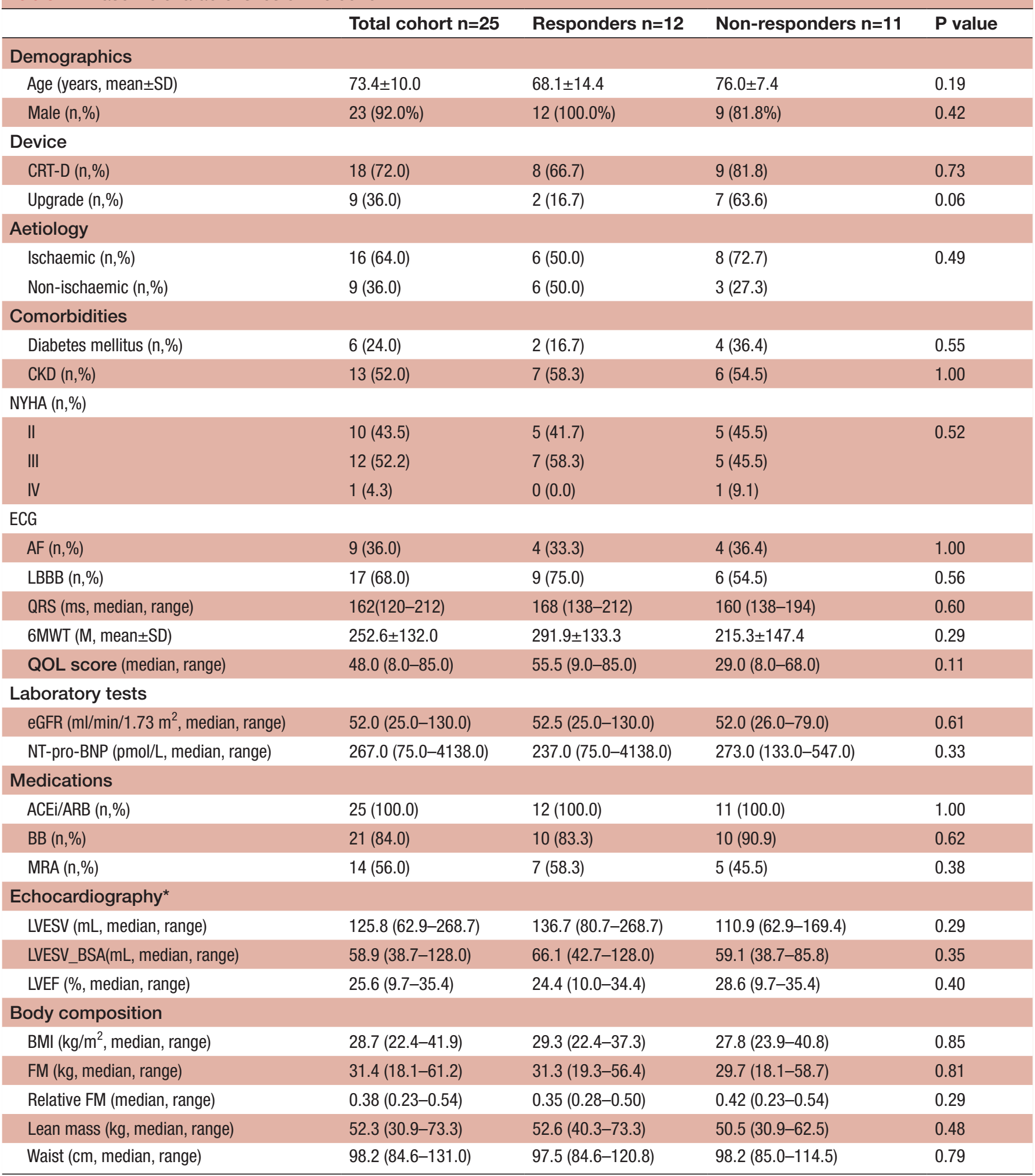

*Based on available data

ACEi, angiotensin converting enzyme inhibitor; AF, atrial fibrillation; ARB, ACE receptor antagonist; BMI, body mass index; BNP, brain natriuretic peptide; BSA, body surface area; CKD, chronic kidney disease; CRT, cardiac resynchronisation therapy; eGFR, estimated glomerular filtration rate; FM, fat mass; LBBB, Left bundle branch block; LVEF, left ventricular ejection fraction; LVESV, left ventricular end systolic volume; 6MWT, 6 min walk test; NT-pro-BNP, N-terminal probrain natriuretic peptide; NYHA, New York Heart Failure Assessment; QOL, quality of life; QRS, QRS width on ECG. 
Table 2 The effect of crt on cardiac function and body composition

\begin{tabular}{|c|c|c|c|c|}
\hline & Baseline & 6 weeks & 6 months & $P$ value \\
\hline QOL score (median, range) & $48.0(8.0-85.0)$ & $33.5(0.0-73.0)$ & $23.5(0.0-0.83)$ & 0.06 \\
\hline 6MWT (M, mean $\pm S D)$ & $252.6 \pm 132.0$ & $277.1 \pm 145.2$ & $242.4 \pm 178.8$ & 0.29 \\
\hline eGFR (ml/min $/ 1.73 \mathrm{~m}^{2}$, median, range) & $52.0(25.0-130.0)$ & $47.0(24.0-105.0)$ & $52.7(20.0-90.0)$ & 0.34 \\
\hline \multicolumn{5}{|l|}{ Body composition (median, range) } \\
\hline BMl (kg/m2) & $28.7(22.4-41.9)$ & $28.5(22.0-34.4)$ & $28.0(22.4-35.7)$ & 0.39 \\
\hline Fat mass $(\mathrm{kg}) \dagger$ & $31.4(18.1-61.2)$ & $29.0(18.9-61.2)$ & $29.1(18.6-50.3)$ & 0.12 \\
\hline Relative fat mass $\dagger$ & $0.38(0.23-0.54)$ & $0.34(0.25-0.51)$ & $0.39(0.28-0.52)$ & 0.09 \\
\hline Lean mass $(\mathrm{kg}) \dagger$ & $52.3(30.9-73.3)$ & $53.9(31.9-75.0)$ & $50.5(31.9-75.0)$ & 0.19 \\
\hline \multicolumn{5}{|l|}{ Echocardiography } \\
\hline LVESV_BSA(mL, median, range) & $58.9(38.7-128.0)$ & $56.8(28.2-118.7)$ & $49.5(30.6-131.5)$ & 0.26 \\
\hline LVEF (\%, median, range) & $25.6(9.7-35.4)$ & $29.4(13.6-43.6)$ & $32.7(14.4-41.7)$ & 0.14 \\
\hline
\end{tabular}

*Based on patients able to have clinical, functional and laboratory assessments (baseline=25, 6 weeks=24, 6 months=22),

†Based on participants able/available to have BOD-POD assessment (baseline=25, 6 weeks $=23,6$ months=20).

$\ddagger$ Based non complete series of echocardiograms with biplane measurements.

$\mathrm{BMI}$, body mass index; BOD POD, trade name of the device used; BSA, body surface area; eGFR, estimated glomerular filtration rate;

LVEF, left ventricular ejection fraction ; LVESV, left ventricular end-systolic volume ; 6MWT, 6 min walk test; NT-pro-BNP, N-terminal

probrain natriuretic peptide; QOL, quality of life.

LVESV/LVESV index were strongly inversely correlated with fat-mass and relative fat mass 6 weeks post-CRT. Fatfree mass (lean mass) decreased post-CRT, fat mass and relative fat mass decreased at first follow-up, returning to baseline at 6 months; this trend was driven primarily by non-responders.

There are currently no data evaluating reliability of ADP in $\mathrm{HF}^{12} \mathrm{ADP}$ interprets body water as fat mass and it is likely that the changes noted may be the result of changes in body water content. Renal function, specifically eGFR, was inversely correlated with change in fat mass at 6 months, trending towards significance at 6-week follow-up.

The association between LV geometry and fat mass relative change following CRT suggests that the increased measured fat mass may be linked to reverse cardiac remodelling. The mechanism for this may be multifactorial and could be related to changes in body water content following CRT. The neurohormonal system may be implicated through reduction in circulating natriuretic peptides in the presence of increased adiposity. ${ }^{13}$ Adipocytes are sensitive to natriuretic peptides, activating lipolysis and enhancing expression of brown adipocyte genes, increasing thermogenesis thereby favouring a procatabolic state in progressive $\mathrm{HF}^{3}$ Naturetic peptides are known to stimulate release of adipokines (specifically adiponectin and leptin), which increase energy utilisation and weight reduction. ${ }^{3}$ Increased NT-pro-BNP immediately after CRT was associated with decreasing fat mass supporting the inverse association between natriuretic peptides and body mass index (BMI) (BMI/fat mass previously reported. ${ }^{36}$ There are limited data on CRT and body composition; a retrospective study of 219 CRT patients evaluated initial BMI predicting response and cardiovascular outcomes at 6 months; they showed overweight/obese patients preimplant had better response and improved outcomes. ${ }^{9}$

Deterioration in renal function and presence of stage 3 chronic kidney disease consistently predicts poor outcomes post-CRT. ${ }^{13}$ Progressive deterioration in eGFR is linked to adverse cardiac remodelling. ${ }^{14}$ The inverse correlation between eGFR and fat mass at 6 months suggests increasing fat mass is associated with deteriorating renal function which is unusual as obesity is believed to preserve renal function. ${ }^{4}$ There was no association between lean mass and BMI. Caution is advised interpreting these results as eGFR is calculated using the renal disease equation, ${ }^{15}$ which uses body surface area as a parameter, meaning eGFR is not an independent variable.

\section{Study limitations}

Our study has several limitations. Our cohort size was small and may impact the findings. However, this was a proof-of-concept pilot study and our findings should generate further studies in larger cohorts. Two of our participants were unable to undergo BOD-POD assessment, which could introduce selection bias. ADP does not account for body water and measures it as fat mass, meaning patient oedema may impact study findings. Our 
study was unable to account for the presence of cardiac cachexia, which is known to be important in advancing HF. ${ }^{1}$ Also, not all patients were able to have complete LV assessment by echocardiography due to body habitus.

\section{CONCLUSION}

This is the first prospective pilot study to examine body composition parameters both before and after CRT implantation. It suggests a trend towards reduction in fat mass in CRT non-responders. Successful CRT response appears to be linked to maintenance of fat mass status at implant. The neurohormonal system appears integral to this.

Acknowledgements We would like to thank the Research, Development and Innovation department at University Hospitals Coventry and Warwickshire for their support with the study.

Contributors All authors contributed to the planning, conduct and reporting of the work described in the article. FO is responsible for the overall content as guarantor.

Funding The authors have not declared a specific grant for this research from any funding agency in the public, commercial or not-for-profit sectors.

Competing interests None declared.

Patient consent for publication Not required.

Provenance and peer review Not commissioned; internally peer reviewed.

Data availability statement Data are available on reasonable request. All data are published in the paper.

Open access This is an open access article distributed in accordance with the Creative Commons Attribution Non Commercial (CC BY-NC 4.0) license, which permits others to distribute, remix, adapt, build upon this work non-commercially, and license their derivative works on different terms, provided the original work is properly cited, appropriate credit is given, any changes made indicated, and the use is non-commercial. See: http://creativecommons.org/licenses/by-nc/4.0/.

ORCID iD

Faizel Osman http://orcid.org/0000-0002-3962-5118

\section{REFERENCES}

1 McAloon CJ, O'Hare P, Osman F, et al. The interplay between heart failure, metabolism and body composition. Br J Hosp Med 2016;77:362-4.

2 Melenovsky V, Kotrc M, Borlaug BA, et al. Relationships between right ventricular function, body composition, and prognosis in advanced heart failure. J Am Coll Cardiol 2013;62:1660-70.

3 Christensen HM, Kistorp C, Schou M, et al. Cross-talk between the heart and adipose tissue in cachectic heart failure patients with respect to alterations in body composition: a prospective study. Metabolism 2014;63:141-9.

4 Clark AL, Fonarow GC, Horwich TB. Obesity and the obesity paradox in heart failure. Prog Cardiovasc Dis 2014;56:409-14.

5 Oreopoulos A, Padwal R, Kalantar-Zadeh K, et al. Body mass index and mortality in heart failure: a meta-analysis. Am Heart $J$ 2008;156:13-22.

6 Loncar G, Bozic B, von Haehling S, et al. Association of adiponectin with peripheral muscle status in elderly patients with heart failure. Eur J Intern Med 2013;24:818-23.

7 Fülster S, Tacke M, Sandek A, et al. Muscle wasting in patients with chronic heart failure: results from the studies investigating co-morbidities aggravating heart failure (SICA-HF). Eur Heart $J$ 2013;34:512-9.

8 Cleland JGF, Daubert J-C, Erdmann E, et al. The effect of cardiac resynchronization on morbidity and mortality in heart failure. $N$ Engl J Med 2005;352:1539-49.

9 Cai C, Hua W, Ding L-G, et al. Association of body mass index with cardiac reverse remodeling and long-term outcome in advanced heart failure patients with cardiac resynchronization therapy. Circ $J$ 2014;78:2899-907.

10 National Institute for Health and Clinical Excellence. Implantable cardioverter defibrillators and cardiac resynchronisation therapy for arrhythmias and heart failure (review of TA95 and TA120), 2014. Available: guidance.nice.org.uk/ta314 [Accessed 14 Jul 2014].

11 Atherton G, McAloon CJ, Chohan B, et al. Safety and costeffectiveness of same-day cardiac resynchronization therapy and implantable cardioverter defibrillator implantation. Am J Cardiol 2016;117:1488-93.

12 Fields DA, Goran MI, McCrory MA. Body-composition assessment via air-displacement plethysmography in adults and children: a review. Am J Clin Nutr 2002;75:453-67.

13 Bogdan S, Klempfner R, Sabbag A, et al. Functional response to cardiac resynchronization therapy in patients with renal dysfunction and subsequent long-term mortality. J Cardiovasc Electrophysiol 2014;25:1188-95.

14 Trespalacios FC, Taylor AJ, Agodoa LY, et al. Heart failure as a cause for hospitalization in chronic dialysis patients. Am J Kidney Dis 2003;41:1267-77.

15 The National Kidney Foundation. Disease outcome Cclinical initiative group. clinical practice guidelines for chronic kidney disease: evaluation, classification, and stratification. Am J Kidney Dis 2002;39:S1-266. 